\title{
Relationship between initial PSA density with future PSA kinetics and repeat biopsies in men with prostate cancer on active surveillance
}

\author{
AF Kotb, S Tanguay, MA Luz, W Kassouf and AG Aprikian \\ Department of Urology, McGill University Health Center, Montreal, Quebec, Canada
}

\begin{abstract}
The objective of our study is to examine the correlation between PSA density (PSAd) at the time of diagnosis with PSA velocity (PSAV), PSA doubling time and tumour progression, on repeat biopsy, in men with prostate cancer on active surveillance. Data from 102 patients with clinically localized prostate cancer on active surveillance in the period between 1992 and 2007, who had the necessary parameters available, were collected. PSAd was calculated and correlated with PSAV, PSA doubling time (PSADT), Gleason score at diagnosis and local progression on repeated biopsies. PSAV was 0.64 and $1.31 \mathrm{ng} \mathrm{ml}^{-1}$ per year $(P=0.02)$, PSADT of 192 and 113 months $(P=0.4)$ for PSAd below and above 0.15 , respectively. The rate of detecting high Gleason score $(\geqslant 7)$ at diagnosis was 6 and $23 \%$ for PSAd below and above 0.15, respectively. A total of 101 patients underwent at least a second biopsy and the incidence of upgrading was 10 and $31 \%$ for PSAd below and above 0.15 , respectively $(P=0.001)$. Although low PSAd is an accepted measure for suggesting insignificant prostate cancer, our study expands its role to indicate that PSAd $<0.15$ may be an additional clinical parameter that may suggest indolent disease, as measured by future PSAV and repeat biopsy over time.

Prostate Cancer and Prostatic Diseases (2011) 14, 53-57; doi:10.1038/pcan.2010.36; published online 12 October 2010
\end{abstract}

Keywords: active surveillance; PSA kinetics; repeat biopsy

\section{Introduction}

There is great disparity between the incidence and mortality of prostate cancer. There is an approximate $15 \%$ risk of men being diagnosed with prostate cancer during their lifetime and only $3 \%$ lifetime risk of death from the disease. ${ }^{1}$ Furthermore, autopsy studies have found that $60-70 \%$ of older men harbour prostate cancer. ${ }^{2}$ Moreover, results from prostate cancer prevention studies ${ }^{3}$ indicate that the rate of finding cancer on biopsy approximates 25\%. Finally, evidence from recently conducted randomized screening trials ${ }^{4}$ suggest that screening for early prostate cancer and subsequent treatment results in improvement in survival, but approximately 48 men require treatment to save one life. Thus, it is clear that more men are being diagnosed with prostate cancer than need be and that active surveillance for early prostate cancer is appealing. The largest experience of active surveillance to date has reported an approximate $30 \%$ progression rate with a median follow-up of 6.8 years. ${ }^{5}$ Several criteria exist in attempting to identify potentially insignificant prostate cancer,

Correspondence: Dr AG Aprikian, Division of Urology, Montreal General Hospital, McGill University Health Centre (MUHC), 1650 Cedar Avenue, Montreal, Quebec, Canada H3G 1A4.

E-mail: Armen.aprikian@muhc.mcgill.ca

Received 10 February 2010; revised 14 June 2010; accepted 21 August 2010; published online 12 October 2010 including serum PSA level, PSA density (PSAd), Gleason score and cancer volume on prostate biopsy. The use of PSAd $<0.15$ as one measure of potentially insignificant prostate cancer is well established. These studies have been conducted on radical prostatectomy specimens where true prostate cancer volume, Gleason score and histological stage were correlated with PSAd at diagnosis.

PSA velocity (PSAV) is one of the important parameters for predicting the behaviour and prognosis of prostate cancer in men undergoing treatment. D'Amico et al. ${ }^{6}$ reported that a greater pretreatment PSAV is associated with a shorter time of disease-specific mortality.

Whether PSAd correlates with future cancer progression, as measured by PSA kinetics or cancer upgrading, is unknown. The aim of our study is to examine the correlation between diagnostic PSAd and subsequent PSA kinetics, serum testosterone and tumour upgrading on follow-up in men with prostate cancer on active surveillance.

\section{Materials and methods}

The McGill University Health Center has been conducting an active surveillance protocol for men with prostate cancer for several years. ${ }^{7}$ Of these men, 102 cases were enrolled in this study based on the availability of complete data. Prostate volume was measured using 
transrectal ultrasound (TRUS) at the time of prostatic biopsy. Prostate volume was measured as a prolate ellipse, calculated as: height $\times$ length $\times$ width $\times \pi / 6$. Width was defined as the maximal transverse diameter at midgland level, length was defined as the distance from the proximal external sphincter to the urinary bladder and height was measured as the midsagittal plane.

All biopsies were retrospectively reviewed by one of two genitourinary pathologists. Considering the biopsy template, we published before ${ }^{7}$ that some of those patients were initially diagnosed by sextant biopsy, whereas others were diagnosed by the current standardized biopsy template of $\geqslant 10$ samples. Repeated biopsies for the whole cohort were done using the current standardized biopsy template for the whole cohort.

Serum PSA measurement used for PSAd was the value observed at presentation before TRUS-guided biopsy. PSAV was calculated using linear regression of all the available PSA data. PSA doubling time was measured using slope regression method of all available PSA values. Gleason score was considered low if it was $\leqslant 6$, and was considered high when it was $\geqslant 7$. Factors correlating with tumour upgrading were studied. Statistical analysis was done using Pearson $\chi^{2}$, Fisher's exact test and Student's $t$-test. Multivariate analysis was done using General Linear Model. A cutoff value for PSAd of 0.15 was used to correlate with PSA kinetics, Gleason score and tumour upgrading. Tumour upgrading was defined as progression from low grade (Gleason $\leqslant 6$ ) to high grade (Gleason $\geqslant 7$ ) or a detection of any new Gleason pattern $\geqslant 4$ on repeat biopsies. Exclusion criteria included patients receiving medications for $\mathrm{BPH}$ in the form of $5 \alpha$-reductase inhibitors, because of their possible effect on serum PSA, prostate volume and serum testosterone measurements.

\section{Results}

The characteristics of the patient cohort are described in Table 1 . The mean age of the patients was 66 years. The mean serum PSA at diagnosis was $5.9 \mathrm{ng} \mathrm{ml}^{-1}$ and the mean PSAd was 0.14. Our patient cohort had a median number of PSA values of 5 (range 3-13). The mean PSAV was $0.88 \mathrm{ng} \mathrm{ml}^{-1}$ per year and the mean PSA doubling time (PSADT) was 172 months. The median number of positive cores was 1 , the median percentage of positive cores was $16 \%$ and the median percentage of tumour volume in the positive cores was $10 \%$. The mean serum testosterone was $12.7 \mathrm{ng} \mathrm{ml}^{-1}$ and the mean level of serum free testosterone was $23.4 \mathrm{pg} \mathrm{ml}^{-1}$. A total of 101 patients included in the study had at least a second biopsy during follow-up and 47, 18 and 7 patients had third, fourth and fifth biopsies, respectively. Of the patients in the study, 90 and 12 were diagnosed with low and high Gleason score cancers, respectively.

The duration of follow-up ranged from 24 to 172 months, with a mean of 61 months. In relation to PSAd, PSAV was 0.64 and $1.31 \mathrm{ng} \mathrm{ml}^{-1}$ per year $(P=0.02)$, PSADT of 192 and 113 months $(P=0.4)$, serum free testosterone 34.2 and $19.5 \mathrm{pg} \mathrm{ml}^{-1}(P=0.0005)$ and serum total testosterone 13.3 and $12.5 \mathrm{ng} \mathrm{ml}^{-1}(P=0.6)$ for PSAd below and above 0.15 , respectively. The rate of detecting high Gleason score at diagnosis was 6 and $23 \%$ for PSAd
Table 1 Patient characteristics

\begin{tabular}{|c|c|}
\hline Mean patient age (years) & $66(49-78)$ \\
\hline \multicolumn{2}{|l|}{ Prostate volume } \\
\hline Mean & $48(10-160)$ \\
\hline Median & 46 \\
\hline Mean serum PSA at diagnosis $\left(\mathrm{ng} \mathrm{ml}^{-1}\right)$ & $5.9(0.18-20.4)$ \\
\hline Mean PSAd at diagnosis & $0.14(0.01-1.3)$ \\
\hline Mean PSAV (ng ml ${ }^{-1}$ per year) & $0.88(0-9.6)$ \\
\hline Mean PSADT (months) & $172(6-500)$ \\
\hline Mean serum total testosterone $\left(\mathrm{ng} \mathrm{ml}^{-1}\right)$ & $12.7(0.4-19)$ \\
\hline Mean serum free testosterone $\left(\mathrm{pg} \mathrm{ml}^{-1}\right)$ & $23.4(0.1-46.9)$ \\
\hline Median number of positive cores & $1(1-6)$ \\
\hline \multicolumn{2}{|l|}{$\%$ of positive cores } \\
\hline Median & $16(10-100)$ \\
\hline$<30 \%$ & 90 \\
\hline$\geqslant 30 \%$ & 12 \\
\hline \multicolumn{2}{|l|}{$\%$ of tumour volume in the positive cores } \\
\hline Median & $10(2-85)$ \\
\hline Number of patients with $<50 \%$ tumour & 94 \\
\hline Number of patients with $\geqslant 50 \%$ tumour & 8 \\
\hline \multicolumn{2}{|l|}{ Gleason score at diagnosis } \\
\hline Low $(<7)$ & 90 \\
\hline High & 12 \\
\hline \multicolumn{2}{|l|}{ Duration of follow-up (in months) } \\
\hline Minimum & 24 \\
\hline Maximum & 172 \\
\hline Mean & 61 \\
\hline \multicolumn{2}{|l|}{ Repeat biopsy } \\
\hline 2 biopsies & 101 \\
\hline 3 biopsies & 47 \\
\hline 4 biopsies & 18 \\
\hline 5 biopsies & 7 \\
\hline
\end{tabular}

Abbreviations: PSAd, PSA density; PSADT, PSA doubling time; PSAV, PSA velocity.

below and above 0.15 , respectively $(P=0.01)$. Of the 101 patients who underwent at least a second biopsy, the rate of upgrading was 10 and $31 \%$ for PSAd below and above 0.15 , respectively $(P=0.001)$. In addition, there was a tendency for cases with PSAd $<0.15$ to present with only a single positive core, whereas cases with PSAd $>0.15$ tended to have more than one positive core of the prostate $(P=0.08)$. Table 2 demonstrates the correlations between PSAd and different variables.

On studying tumour upgrading, most of the cases that showed upgrading had their Gleason score change from $\leqslant 6$ to $>6$, except two cases with initial Gleason score 7 $(4+3)$ that developed new patterns 4 and 5, changing their score to 8 and 9 . Both PSAd and PSAV significantly correlated with tumour upgrading on univariate analysis, whereas only PSAd remained to be independent significant factor predicting progression on multivariate analysis. Table 3 demonstrates univariate analysis of factors predicting tumour upgrading on repeated biopsy. Multivariate analysis was performed (Table 4), where PSAd represents PSAd with a cutoff value of 0.15 and PSAV represents PSAV with a cutoff value of 0.75 . Multivariate analysis showed PSAd to be an independent factor for predicting tumour upgrading on repeat biopsy, whereas it showed a strong trend to predict PSAV on follow-up. Twenty-five patients had PSAd ranging 
Table 2 PSA density at diagnosis and PSA kinetics and repeat biopsy over time

\begin{tabular}{lccc}
\hline PSAd & $\leqslant 0.15$ & $>0.15$ & P-value \\
\hline PSAV & 0.64 & 1.31 & 0.02 \\
PSAV & & & \\
$\quad \leq 0.75$ & 47 & 17 & 0.02 \\
$>0.75$ & 20 & 18 & \\
PSADT & 192 & 113 & 0.4 \\
Gleason & & & \\
$\quad$ Low & 63 & 27 & 0.01 \\
High & 4 & 8 & \\
& & & \\
Positive cores & 47 & 19 & 0.08 \\
$\quad$ core & 20 & 16 & \\
$\quad>1$ & 13.3 & 12.5 & 0.6 \\
Total testosterone & 34.2 & 19.5 & 0.0005 \\
Free testosterone & & & \\
Repeat biopsy & 59 & 24 & 0.001 \\
$\quad$ Same grade & $7(10 \%)$ & $11(31 \%)$ & \\
$\quad$ Upgrading & & & \\
\hline
\end{tabular}

Abbreviations: PSAd, PSA density; PSADT, PSA doubling time; PSAV, PSA velocity.

Table 3 Univariate analysis of factors predicting tumour upgrading

\begin{tabular}{lrrr}
\hline & \multicolumn{2}{c}{ Tumour upgrading } & P-value \\
\cline { 2 - 3 } & No & Yes & \\
\hline PSA & & & \\
$\quad \leqslant 10$ & 77 & 17 & 0.8 \\
$>10$ & 6 & 1 & 0.05 \\
$\leqslant 4$ & 20 & 1 & \\
$>4$ & 63 & 17 & \\
PSAd & & & \\
$\quad \leqslant 0.15$ & 58 & 8 & \\
$>0.15$ & 25 & 10 & 0.03 \\
& & & \\
PSAV & & 7 & \\
$\quad \leqslant 0.75$ & 56 & 11 & \\
$>0.75$ & 27 & & \\
Positive cores & & 16 & \\
$<3$ & 74 & 2 & \\
$\geqslant 3$ & 9 & & \\
\hline
\end{tabular}

Abbreviation: PSAd, PSA density; PSAV, PSA velocity.

from 0.13 to 0.17 , of which only five showed upgrading on repeated biopsy. None of those five patients had PSAd $<0.15$. One patient had PSAd of exactly 0.15 , two patients of 0.16 and two patients of 0.17 .

Interestingly, serum PSA at diagnosis was not a significant factor predicting PSAV, although it was of significance predicting tumour upgrading. Studying the changes of PSAd over time during repeated biopsy, it was clear that there is a tendency for each group of patients with PSAd $\leqslant 0.15$ and $>0.15$ to remain in their initial PSAd group. However, of patients with initial PSAd $>0.15$ (35 patients), we had three patients $(8.5 \%)$ that showed a drop of PSAd to $\leqslant 0.15$. Their drop in
Table 4 Multivariate analysis

\begin{tabular}{ll}
\hline Variables & P-value \\
\hline PSAV $\left(0.75 \mathrm{ng} \mathrm{ml}^{-1}\right.$ per year $)$ & \\
PSAd & 0.06 \\
PSA & 0.5 \\
Gleason grade & 0.09 \\
Gleason score at diagnosis & \\
PSAd & 0.01 \\
PSA & 0.2 \\
PSAV & 0.09 \\
Tumour upgrading on repeat biopsy & \\
PSAd & 0.008 \\
PSAV & 0.1 \\
PSA & 0.01 \\
Gleason grade & 0.08 \\
\hline
\end{tabular}

Abbreviations: PSAd, PSA density; PSAV, PSA velocity.

Table 5 PSAd changes during follow-up

\begin{tabular}{llc}
\hline Initial PSAd & \multicolumn{2}{c}{ Follow-up PSAd } \\
\cline { 2 - 3 } & \multicolumn{1}{c}{$<0.15$} & $>0.15$ \\
\hline$<0.15$ (66 patients) & 56 patients (85\%) & 10 patients (15\%) \\
$>0.15$ (35 patients) & 3 patients (8.5\%) & 32 patients $(91.5 \%)$ \\
\hline
\end{tabular}

Abbreviation: PSAd, PSA density.

PSAd can be explained perhaps by increased prostate volume over time (follow-up of 24 months). Two patients had initial Gleason score of 6 that inspite of a change in PSAd into lower category due to increased prostate volume they still showed upgrading into Gleason score of 7. The third patient had initial Gleason score of 7 $(3+4)$ that did not change during follow-up. On the other hand, of the patients with initial PSAd $\leqslant 0.15$, we had 10 patients $(15 \%)$ that showed an increase in their PSAd into $>0.15$ category during follow-up, which can perhaps be attributed to the higher PSAV in that group (median PSAV =1). Tables 5 shows PSAd measurements changes over time. A Supplementary Table is available online showing the results of prostate volume measurements for each patient during follow-up.

\section{Discussion}

Active surveillance is an established option for the management of selected men with localized prostate cancer. $^{5}$ Both the European ${ }^{8}$ and the National Comprehensive Cancer Network ${ }^{9}$ guidelines recommend the use of PSAV in the follow-up of cases on active surveillance and consider higher PSAV to be a sign of progression that require treatment. The National Comprehensive Cancer Network defined PSAV $>0.75$ to be a sign of progression for prostate cancer for cases on active surveillance, whereas the European guidelines did not define a value of PSAV indicative of progression. Many studies 8,10 have found a correlation between initial serum PSA and future PSAV, in which high initial serum PSA was found to be associated with high PSAV and 
higher incidence of tumour upgrading. Our study did not find a correlation between initial serum PSA and PSAV on follow-up, although it was of significant value in predicting tumour upgrading on repeat biopsy.

However, PSAd at the time of the diagnosis of prostate cancer with subsequent PSAV showed a significant correlation, as PSAd $<0.15$ was associated with a lower PSAV (mean of 0.64), whereas PSAd >0.15 was associated with a markedly higher PSAV (mean of 1.31). Furthermore, higher PSAd correlated independently with greater rates of tumour upgrading on repeat biopsy with $31 \%$ for cases with PSAd $>0.15$. Conti et al. ${ }^{11}$ estimated an upgrading rate for cases suitable for active surveillance of $28 \%$. Smaldone et al. ${ }^{12}$ demonstrated a rate of upgrading reaching $27 \%$ for cases suitable for active surveillance. In our cohort, we had a similar rate of upgrading for cases with PSAd $>0.15$, whereas significantly much lower rate of upgrading (10\%) for cases with PSAd $\leqslant 0.15$. Of course PSAd requires prostate volume measurement, which can be variable by TRUS Although the ellipsoid formula is not perfect, some ${ }^{13,14}$ have confirmed its accuracy for estimating prostate volume and its close correlation with pathological prostatic volume. To date, the literature supports the use of TRUS-based prostate volume measurements, and as it is now used routinely to conduct prostate biopsy, we feel that measuring prostate volume using TRUS to determine PSAd remains a clinically relevant tool. Our analysis showed that in our cohort there was a mean increase in prostate volume of $10 \%$ over time, whether this is variability of TRUS volume measurements or an actual increase over time is unknown. The tendency to keep within the same PSAd group during follow-up is another factor confirming the accuracy of our prostate volume measurements, where the majority of patients within initial PSAd group tended to remain within the same group during a mean follow-up of 5 years.

Although we observed a trend towards longer PSADT in cases of low PSAd, this did not achieve statistical significance. Some recent studies ${ }^{15,16}$ investigated the use of PSAV and PSADT on the same group of patients to predict high-risk prostate cancer and concluded that PSAV is a more useful parameter than PSADT to identify men with life-threatening disease.

Out of our 18 patients that developed tumour upgrading, 10 patients were managed by radical prostatectomy, 4 patients by external beam radiation, 2 patients by brachytherapy and 2 patients refused intervention and kept on active surveillance. Gleason score following radical prostatectomy was persistent with the biopsy findings.

There is accumulating literature suggesting an association between low serum testosterone with prostate cancer. Yamamoto et al. ${ }^{17}$ detected an association between low serum testosterone at presentation with positive surgical margins following radical prostatectomy and worsening overall survival. Morgentaler ${ }^{18}$ demonstrated the association between low serum testosterone with higher Gleason grade cancer. In addition, Nishiyami et al. ${ }^{19}$ reported that intraprostatic dihydrotestosterone levels were significantly reduced in men with Gleason $\geqslant 7$ cancer compared with men with Gleason score $\leqslant 6$. Based on these studies, we decided to also examine the relationship of serum testosterone with PSAd in our cohort of patients on active surveillance. Interestingly, we observed a similar relationship in that in cases with higher PSAd $(>0.15)$ there was a significantly lower serum free testosterone and vice versa.

\section{Conclusion}

PSAd appears to be a reliable clinical parameter to predict the behaviour of clinically localized prostate cancer on active surveillance. Our data suggests that cases with PSAd $<0.15$ can be followed up safely on active surveillance, whereas cases with PSAd $>0.15$ are at a higher risk of tumour progression and may be better managed by definitive therapy.

\section{Conflict of interest}

The authors declare no conflict of interest.

\section{References}

1 Jermal A, Siegel R, Ward E, Murray T, Xu J, Smigal C et al. Cancer statistics, 2006. CA Cancer J Clin 2006; 56: 106.

2 Sakr WA, Grignon DJ, Crissman JD, Heilbrun LK, Cassin BJ, Pontes JJ et al. High grade prostatic intraepithelial neoplasia (HGPIN) and prostatic adenocarcinoma between the ages of 2069: an autopsy study of 249 cases. In Vivo 1994; 8: 439.

3 Thompson IM, Ankerst DP, Chi C, Goodman PJ, Tangen CM, Lucia MS et al. Assessing prostate cancer risk: results from the prostate cancer prevention trial. J Natl Cancer Inst 2006; 98: 529-534.

4 Schroder FH, Hugosson J, Roobol MJ, Tammela TL, Ciatto S, Nelen $\mathrm{V}$ et al. Screening and prostate cancer mortality in a randomized European study. N Eng J Med 2009; 360: 1320.

5 Klotz L, Zhang L, Lam A, Nam R, Mamedov A, Loblaw A. Clinical results of long term follow up of a large active surveillance cohort with localized prostate cancer. J Clin Oncol 2010; 28: 126-131.

6 D'Amico A, Chen M, Roehl K, Catalona WJ. Preoperative PSA velocity and the risk of death from prostate cancer after radical prostatectomy. N Engl J Med 2004; 351: 125-135.

7 Al Otaibi M, Ross P, Fahmy N, Jeyaganth S, Trottier H, Sircar K et al. Role of repeated biopsy of the prostate in predicting disease progression in patients with prostate cancer on active surveillance. Cancer 2008; 113: 286-292.

8 Van den Bergh R, Roemeling S, Roobol MJ, Wolters T, Schroder $\mathrm{FH}$, Bangma $\mathrm{CH}$. Prostate specific antigen kinetics in clinical decision making during active surveillance for early prostate cancer: a review. Eur Urol 2008; 54: 505-516.

9 NCCN. NCCN clinical practice guidelines in oncology (2007) prostate cancer early detection. Version 2. Accessed at http:// www.nccn.org/professionals/physician_gls/PDF/prostate.pdf

$10 \mathrm{Yu}$ X, Loeb S, Roehl KA, Han M, Catalona WJ. The association between total PSA concenteration and prostatic specific antigen velocity. J Urol 2007; 177: 1298-1302.

11 Conti SL, Dall'era M, Fradet V, Cowan JE, Simko J, Carroll PR. Pathological outcomes of candidates for active surveillance of prostate cancer. J Urol 2009; 181: 1628-1633.

12 Smaldone MC, Cowan JE, Carroll PR, Davies BJ. Eligibility for active surveillance and pathological outcomes for men undergoing radical prostatectomy in a large, community based cohort. J Urol 2010; 183: 143-144.

13 Eri LM, Thomassen H, Brennhovd B, Håheim LL. Accuracy and repeatability of prostate volume measurements by transrectal ultrasound. Prostate Cancer Prostatic Dis 2002; 5: 273-278.

14 Park SB, Kim JK, Choi SH, Noh HN, Ji EK, Cho KS. Prostate volume measurement by TRUS using heights obtained by transaxial and midsagittal scanning: comparison with specimen 
volume following radical prostatectomy. Korean J Radiol 2000; 1: $110-113$.

15 Loeb S, Kettermann A, Ferrucci L, Landis P, Metter EJ, Carter HB. PSA doubling time versus PSA velocity to predict high-risk prostate cancer: data from the Baltimore Longitudinal Study of Aging. Eur Urol 2008; 54: 1073-1080.

16 Ng MK, Van As N, Thomas K, Woode-Amissah R, Horwich A, Huddart $\mathrm{R}$ et al. Prostate-specific antigen (PSA) kinetics in untreated, localized prostate cancer: PSA velocity vs PSA doubling time. BJU Int 2009; 103: 872-876.

17 Yamamoto S, Yonese J, Kawakami S, Ohkubo Y, Tatokoro M, Komai $\mathrm{Y}$ et al. Preoperative serum testosterone level as an independent predictor of treatment failure following radical prostatectomy. Eur Urol 2007; 52: 696-701.
18 Morgentaler A. Testosterone deficiency and prostate cancer: emerging recognition of an important and troubling relationship. Eur Urol 2007; 52: 623.

19 Nishiyama T, Ikarashi T, Hashimoto Y, Suzuki K, Takahashi K. Association between dihydrotestosterone level in the prostate and prostate cancer aggressiveness using the Gleason score. J Urol 2006; 176: 1387-1391.

This work is licensed under the Creative Commons Attribution-NonCommercial-No Derivative Works 3.0 Unported License. To view a copy of this license, visit http://creativecommons.org/licenses/ by-nc-nd/3.0/

Supplementary Information accompanies the paper on the Prostate Cancer and Prostatic Diseases website (http:// www.nature.com/pcan) 